Trabajos y Comunicaciones, 2da. Época, No 46, e041, octubre 2017. ISSN 2346-8971

Universidad Nacional de La Plata.

Facultad de Humanidades y Ciencias de la Educación.

Departamento de Historia

\title{
Violencia institucional y represión paraestatal. Algunas reflexiones a partir del caso mendocino 1
}

\author{
Institutional violence and para statal repression. Some reflections \\ from the Mendoza case
}

\section{Laura Rodríguez Agüero *}

* Instituto de Ciencias Humanas Sociales y Ambientales-CONICET / Universidad Nacional de Cuyo, Argentina | Irodriguezaguero@gmail.com

\section{PALABRAS CLAVE \\ Represión}

Violencia institucional

Historia reciente

\section{RESUMEN}

En esta comunicación nos proponemos esbozar las primeras pinceladas sobre uno de los aspectos poco explorados de la represión previa al golpe de Estado de 1976: la persecución y asesinato de lo que la prensa de la época denominaba “delincuentes comunes”. A través del análisis de diarios se explica de qué manera estas muertes relacionadas con los negocios policiales actuaron y se articularon con la trama represiva que estaba siendo puesta en marcha en ese momento. El estudio se centrará en el caso mendocino entre los años 1974 y 1976.

\section{KEYWORDS}

\section{ABSTRACT}

Repression In this communication we propose to outline the first brushstrokes about one of the unexplored aspects of the repression prior to the coup d'état of 1976: the persecution and

Institutional violence murder of what the press of the time called "common delinquents." Through the analysis of

Recent history newspapers it is explained how those deaths related to the police business acted and were articulated with the repression network that was being implemented at that time. The study will focus on the Mendoza case between 1974 and 1976. 
I.

La represión llevada adelante por la última dictadura cívico militar, tuvo un carácter multiforme que en los últimos años se ha ido complejizando. La idea inicial de un "enemigo interno" identificado estrictamente con militantes políticos-sindicales, junto con la idea de perpetradores vinculados sólo a las fuerzas armadas y de seguridad así como una periodización rígida que marcaba el comienzo del terror en marzo del 76, se fueron ampliando para dar lugar a nuevos estudios que han visibilizado nuevas aristas de la dinámica represiva, desconocidas años atrás² .

Siguiendo a Gabriela Águila quien plantea que las variaciones regionales en la implementación de la represión fueron tan importantes como la sistematicidad del accionar represivo, en las investigaciones que he llevado a cabo sobre la provincia de Mendoza he prestado atención a dichas variaciones, encontrando elementos novedosos en relación a estudios realizados en otros lugares del país (Águila, 2013).

De manera sintética e ilustrativa podemos señalar que en Mendoza se combinaron diversos factores en el desarrollo de la estrategia represiva. Entre 1973 y 1976, en un marco de creciente conflictividad social, de desmoronamiento del Pacto Social, de crisis institucional y económica, se puso en marcha un dispositivo represivo que a nivel provincial actuó a través del CAM (Comando Anticomunista Mendoza) y del Comando Moralizador Pío XII. Ambos comandos, que fueron la expresión "política”, en el primer caso, y “moralizadora” en el segundo, de la violencia paraestatal a nivel local, eran parte de una misma organización encabezada por el jefe de policía vicecomodoro J. C. Santuccione, quien fuera enviado por J. López Rega a la provincia. Su accionar, dejó como saldo cientos de atentados con bombas, secuestros y asesinatos. En trabajos anteriores analizamos la naturaleza del accionar represivo y dimos cuenta de los dispositivos utilizados por los comandos mencionados, partiendo de la idea de que la magnitud que la violencia paraestatal adquirió en Mendoza se debió, por un lado, a la existencia de una fuerte tradición nacionalista de derecha en la provincia, que confluyó en el combate a la denominada "subversión"; y por otro, al tejido de redes represivas en el Cono Sur que se configuró hacia mediados de la década del 70 (Rodriguez Agüero, 2012 y 2013b). Otro rasgo destacado de la represión pre dictatorial en la provincia fue el carácter "sexuado" que la misma adquirió. En este punto, la perspectiva de género nos permitió visualizar cómo los casos de secuestro, tortura y asesinato de mujeres en situación de prostitución tuvieron un carácter claramente político. Este hecho nos llevó a reconsiderar la idea de "enemigo interno", habitualmente asociada -y limitada- a militantes (gremiales o partidarios) ligados a la izquierda marxista o peronista (Rodriguez Agüero, 2013a).

En esta comunicación nos proponemos esbozar las primeras pinceladas sobre otro de los aspectos poco explorados de la represión previa al golpe de Estado de 1976: la persecución y asesinato de lo que los diarios de la época denominaban "delincuentes comunes”. Esta inquietud surgió cuando, al realizar el relevamiento de diarios con el objetivo de reconstruir el accionar represivo paraestatal en Mendoza en los meses previos a la dictadura, nos encontramos con numerosos casos de asesinatos de "delincuentes comunes” 3 . Es decir, que entre las noticias que daban cuenta de la brutalidad de un aparato represivo que "se estaba poniendo a punto" y que se cobraba la vida de numerosos militantes populares, aparecían también crónicas que relataban cómo jóvenes de sectores populares, vinculados la mayor parte de las veces a negocios ilegales como la quiniela, las drogas o la prostitución, aparecían asesinados en los mismos lugares y con los mismos métodos que las y los primeros militantes víctimas del terror. Estos hallazgos nos llevaron a introducir este novedoso factor al análisis, y a preguntarnos de qué manera estas muertes relacionadas con los negocios policiales actuaron y se articularon con la trama represiva que estaba siendo puesta en marcha en ese momento. Al ser un trabajo inicial, en esta comunicación apelaremos sólo a fuentes periodísticas de la época y plantearemos más interrogantes que respuestas.

\section{La persecución de la "delincuencia común”}

Sofía Tiscornia señala que la violencia policial en Argentina tiene larga data y hace hincapié en la importancia de analizar la "zona opaca de la violencia de policía" es decir, aquella en que dicha violencia aparece como una cuestión normalizada, naturalizada. Esa "zona opaca a la mirada pública”, señala la autora, "es aquella en la que 
los muertos por la policía se han convertido en "abatidos”, en personajes a los que -se dice- la mala vida les ha escrito con desmesura frondosos prontuarios. Individuos que son sólo bajas en batallas cuyos partes se comunican todos los días en policiales de diarios y noticieros” (Tiscornia 2008: 2)

¿Quiénes son las víctimas de estas prácticas violentas? La autora las caracteriza de la siguiente manera:

“jóvenes y adolescentes que habían traicionado los pequeños negocios de la ilegalidad policial; que se negaban a ser "buchones"; que estaban en la esquina del barrio cuando el patrullero los confunde con otros de quienes querían vengarse, o pobres diablos engañados para lo que sería luego la escenificación de un falso "operativo" policial, obviamente exitoso. Todas esas muertes eran muertes naturalizadas (Tiscornia 2008: 2)”

Si bien el acceso a fuentes es escaso, de hecho sólo contamos con diarios de la época, haremos un intento por abordar algunos de esos casos ocurridos en el candente clima pre dictatorial mendocino. Para ejemplificar la magnitud de esta situación, daremos algunas cifras aproximadas y provisorias producto de investigaciones de los organismos de DDHH, de la justicia y propias. En 1974 se registran 4 muertes de personas ligadas a la prostitución. Entre enero de 1975 y el 24 de marzo de 1976 sabemos de 16 asesinatos vinculados a "negocios ilegales” (8 NN y 8 que fueron identificados) y otros 28 casos, entre asesinatos y desapariciones, por razones políticas; lo que implica un total aproximado -y provisorio- de 60 personas muertas y/o desaparecidas producto de la violencia estatal / paraestatal.

Las víctimas que no tenían relación con ningún tipo de militancia política, social, barrial, sindical, etc., entran en la caracterización hecha por Tiscornia: eran personas ligadas a negocios ilegales de la policía, principalmente jóvenes de los barrios periféricos de la ciudad de Mendoza. A continuación nombraremos algunos de esos casos: una mujer de 35 años, su cuerpo calcinado es hallado en Canota; un hombre no identificado que presenta impactos de bala y las manos quemadas; Alfredo Zabaleta, de 25 años, quien había sido detenido por averiguación de antecedentes. Su madre lo buscaba desde el momento en que había salido en libertad de la seccional Tercera (Los Andes, 4/05/1975). Margarita Albornoz, mujer en situación de prostitución quien fue asesinada y su cuerpo hallado el 12 de abril de 1974 en Canota; Felix “Nene” Morales y Jose "Pepe” Morabito quienes horas antes de su desaparición, a comienzos de 1975, habían estado detenidos en Unidad Regional Primera. Sus cuerpos calcinados aparecieron en Papagallos (Los Andes, 6/05/1975). También Roberto Lascano de 31 años, “ex ladrón de autos”; dos mujeres en situación de prostitución Claridad Gonzalez y Ramona Suarez, encontradas el 1/05/1975 desnudas (con signos de violación y tortura) y con disparos en sus cabezas, habían sido secuestradas la noche anterior por un comando; Gaspar Aguilera acribillado en Papagallos, quien había estado preso por hurto y "trata de blancas”; Miguel Ángel Sanchez “el cachorro chico” acribillado en San Isidro, había estado detenido también por averiguación de antecedentes, entre otros (Los Andes, 6/05/1975). A todos ellos se sumaban otros cadáveres que aparecieron calcinados en los mismos parajes montañosos pero que nunca fueron identificados (quedaron como NN).

Las noticias de estos asesinatos aparecían de manera paralela a las de militantes asesinados/as, sólo que tenían menor repercusión. A medida que el año 1975 avanzaba, y se agudizaba la represión paraestatal sobre militantes vinculados a la izquierda peronista y marxista, las cifras de asesinatos de jóvenes de sectores populares por parte de las policías también se disparaba, lo cual nos hace pensar que estos hechos, lejos de ser casos aislados, forman parte de un fenómeno mayor íntimamente relacionado al accionar de las fuerzas de seguridad.

A continuación mencionaremos tres casos que nos permiten comenzar a escarbar en las inquietudes aquí propuestas.

El primero de ellos es el caso del secuestro y tortura del joven Ricardo Benítez. En noviembre de 1975, se publica la noticia sobre la desaparición de este estudiante, cuya madre había ido a pedir colaboración al diario Los Andes. El joven de 21 años había sido secuestrado en su lugar de trabajo el mediodía del 19 de noviembre, en el centro de la ciudad. Su madre relataba a Los Andes: “Llegaron a su lugar de trabajo por lo menos cuatro individuos (...) uno 
de los extraños mostró su credencial y dijo ser policía” (Los Andes 22/11/1975). Ya en estas denuncias el diario se refiere a los secuestrados como "desaparecidos" a la vez que da cuenta del peregrinaje de los familiares por comisarías, lugares en los que los oficiales de turno dicen no tener ningún registro de lo acontecido. El segundo caso es el del joven Hugo Alaniz, quien fue detenido días antes del golpe de Estado, por agentes de civil durante un baile de carnaval y falleció producto de las torturas a las que fue sometido en el Centro Clandestino de Detención (CCD), D-2. Decía Los Andes en su crónica del 19 de marzo "la denuncia la formuló la madre de la víctima y según su testimonio, como la de otros testigos y conforme surgiría de las declaraciones formuladas por Alaniz a dos médicos que lo atendieron, habría sido sometido a apremios ilegales por efectivos policiales en el Palacio Policial” (Los Andes 19/03/2017).

La nota continuaba: "De acuerdo al cúmulo de relatos acopiado por el periodismo, todo se habría iniciado en el baile del domingo pasado en el estadio Pacifico". Un testigo que se encontraba con Alaniz en ese momento informaba que habían llegado cinco hombres de civil diciendo que estaban buscando al joven. La madre a su vez señalaba que "los sujetos se identificaron como pertenecientes a la sección Robos y Hurtos de la Dirección de Investigaciones” (Los Andes, 19/03/2017). El mismo testigo, cuya identidad no fue revelada por los periodistas por una cuestión de seguridad, comentó que fueron llevados al Palacio Policial y que pudieron escuchar los gritos de Alaniz cuando era sometido a tortura con picana eléctrica. Según el mismo Alaniz alcanzó a comentar a sus amigos, le querían adjudicar un robo en Palmira, pero él se negó en todo momento. Luego el joven fue liberado y llegó a su casa con fuertes dolores abdominales por lo que fue llevado al hospital Central, donde falleció.

Como en otros casos, su madre y su tía se presentaron a Los Andes a radicar la denuncia, a raíz de lo cual periodistas del diario se dirigieron primero a entrevistar al médico que lo había atendido en el hospital Central, y luego a la jefatura de Policía donde increparon al sub jefe "quien se mostró sorprendido y dijo no conocer el caso pero en presencia de los cronistas ordenó -telefónicamente- una amplia investigación para determinar si había responsabilidad policial" relataba la crónica del 19 de marzo . Vale la pena recalcar que Alaniz estuvo secuestrado y fue torturado en el Palacio Policial donde funcionó el D2, principal centro clandestino de detención de la provincia. Este joven, a diferencia de Benítez de quien no conocemos cual fue su destino, está incluido en la lista de asesinados-as y detenidos-as desaparecidos-as de Mendoza.

Otro de los casos ilustrativos es el del ciudadano chileno Juan Hidalgo, quien había sido secuestrado y torturado por la policía, que a continuación lo había llevado a Papagallos, donde había sido baleado y abandonado. Hidalgo fingió estar muerto y logró llegar hasta la ruta donde paró un camión que lo llevó herido al hospital Central, ubicado en la ciudad de Mendoza. El jefe de policía Santuccione negó el hecho por lo que periodistas de Los Andes y El Andino emprendieron su propia investigación. Al respecto el 13 de enero de 1976 Los Andes señalaba:

"las expectativas creadas en torno a un grave episodio ocurrido el viernes último (...) resultaron satisfechas ayer cuando redactores de Los Andes y El Andino pudieron llegar hasta la víctima del hecho, conocer detalles inéditos hasta ese momento y obtener un comunicado de la jefatura de la policía (Los Andes, 23/2/1976).”

En una nota anexa publicada el mismo día, explicaban que "en los últimos tres días había sido imposible ubicar el lugar adonde era asistida la víctima por lo que la investigación periodística había llegado a un límite” (Los Andes, 23/2/1976). Luego relataban cómo obtuvieron la pista de que podría estar en el hospital Central, por lo que se dirigieron ahí con un fotógrafo burlando las guardias policiales. Los cronistas lograron mantener un mínimo diálogo con la víctima, comprobando que efectivamente era Hidalgo y que la policía había intentado asesinarlo. El breve diálogo terminó cuando dos policías los descubrieron “y los sacaron sin violencias pero con energía” (Los Andes, 23/2/1976). El fotógrafo alcanzó a registrar la imagen del hombre baleado. Luego de la intervención periodística, el jefe de Policía debió asumir el hecho y justificar el ocultamiento de la información diciendo que "se había querido llevar la investigación con el máximo secreto posible a fin de que no quedaran vacíos en la pesquisa y tenerse la seguridad de que los culpables serían detenidos” (Los Andes, 23/2/1976). Este hecho tuvo 
gran repercusión, los policías implicados fueron pasados a retiro y ante rumores de que la policía sería intervenida, el gobernador-interventor Lucero debió salir a respaldar a Santuccione a través de una declaración pública.

\section{Moral, negocios policiales y Terror sobre las “clases peligrosas”}

¿Qué tienen en común estos casos que nos lleva a enmarcarlos como parte de la violencia institucional que se articuló con la violencia paraestatal previa al golpe?

En todos ellos se observa un mismo modus operandi que es el mismo al que fueron sometidos los y las militantes: secuestros seguidos de torturas, muertes violentas, los cadáveres presentan impactos de bala, muchos con las manos o el cuerpo calcinados, los cuerpos son arrojados en los mismos lugares de montaña (Canota, Papagallos y San Isidro).

Según las crónicas periodísticas, las víctimas aparecen muertas luego de haber sido detenidas por la policía y todos ellos pertenecen a sectores populares. Sofía Tiscornia señala que los hechos de violencia policial “son aplicados fundamentalmente sobre aquellos grupos o personas que, pertenecientes a sectores pobres, transitan el ambiguo y anchuroso espacio de la ilegalidad” (Tiscornia 2008). En este caso, gran parte de ellos estaban implicados en quinielas clandestinas, prostitución o robos. Los asesinos fueron claramente efectivos de la policía de Mendoza y en el caso de Alaniz, quien fue asesinado por negarse a adjudicarse un robo, fue torturado en el CCD D2, lugar que en ese momento ya era usado para el cautiverio de personas secuestradas por motivos políticos.

Respecto de la participación de la policía en acciones violentas, encontramos también que parte de las operaciones llevadas adelante por el Comando Moralizador Pío XII, y que consistían en atentados con bombas a clubes nocturnos (además del asesinato de mujeres en situación de prostitución) estaban ligadas más que a una cuestión de "saneamiento moral”, a negocios ilegales que comprometían a las fuerzas de seguridad. En esa dirección contamos con un testimonio que da cuenta del "negocio” que implicó para esa época el pago de coimas a cambio de “ser protegidos del Comando”. Comenta Alberto Atienza, editor de policiales del diario Mendoza durante esos años:

“El Comando tenía dos líneas, una era una supuesta defensa de la moral y las buenas costumbres y otra económica, de extorsión (...) Esta segunda línea consistía en ir a lugares de la noche, que todo el mundo sabe, se maneja mucho dinero y pedir cierta cantidad a cambio de seguridad. Uno de esos cabarets era el famoso La Noche, ahí había un griego, al que le piden dinero y no quiere pagar la cantidad que pedían y estalla una bomba. Ocurre algo similar con otro boliche Barrabás, de Néstor Sierra. Me cuenta ese hombre que habían ido dos hombres a verlo y me consulta que podía hacer y le dije, si podés, pagales, pero explicales que es por única vez, que hasta allí te alcanza. Les pagó y nunca le pasó nada” (Atienza, marzo de 2008).

En este punto nos preguntamos; ¿de qué modo se imbricaron las ideas del delincuente común con la del delincuente subversivo en este contexto?. Al respecto Tiscornia señala

“La categoría de delincuente no incluye solamente a aquellas personas que atentan contra la vida o propiedad de otras. Es justamente su fuerte dependencia del poder político la que resulta en que, según las circunstancias, disidentes y opositores políticos o grupos que defienden sus derechos sean considerados en la misma categoría de "delincuentes - peligrosos - enemigos”. Y, en general, todos aquellos que puedan homologarse con las "clases peligrosas” según las épocas: migrantes, mendigos, pobres, vendedores ambulantes; o que expresan diferencias: homosexuales, travestis (Tiscornia, 2008:6).”

Este señalamiento realizado por Tiscornia nos permite comenzar a echar luz sobre la vinculación entre el 
acelerado crecimiento de asesinatos y desapariciones protagonizados por las fuerzas de seguridad en los momentos previos al golpe de Estado, y al modo en que en un contexto de violencia paraestatal, se homologó a “delincuentes subversivos” con “delincuentes comunes y prostitutas”. Y acá nos interesa destacar que si bien sabemos que la violencia institucional es un fenómeno de larga data, en momentos de montaje del dispositivo represivo esas prácticas violentas tomaron la misma forma que las acciones “antisubversivas” en cuanto al modo en que fueron llevadas adelante. El arrojar en Papagallos, Canota o San Isisdro cuerpos con signos de tortura y calcinados, de personas que habían sido detenidas o secuestradas por la policía, se transformó en algo habitual durante esos años, fueran militantes o quinieleros. Incluso el hostigamiento de mujeres en situación de prostitución, que tampoco era un fenómeno nuevo, en los momentos previos al golpe tomó características novedosas (Rodriguez Agüero, 2013a). Por otra parte, el número de asesinatos que se podría incluir dentro de lo que denominamos violencia institucional creció notablemente a medida que el aparato terrorista paraestatal acrecentaba sus acciones.

Un último elemento que queremos mencionar, tiene que ver con el manto moralizador que la misma policía impuso a muchas de sus prácticas violentas. Para ello traeremos a colación solo dos ejemplos para ilustrar esta situación, y también para mostrar como lo ilegal y lo legal se articularon a la hora de aplicar la estrategia represiva.

En primer lugar, señalar, cómo la persecución ilegal de carácter “moral” y violenta de mujeres en situación de prostitución se combinó con medidas legales tales como la reforma del código de faltas. Por aquellos años se había presentado un proyecto para modificar el Código de Faltas que incluía el castigo a la "Prostitución

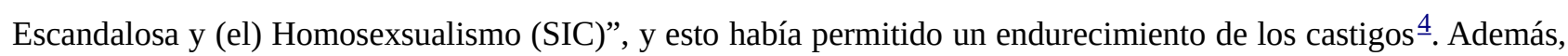
bajo la idea de que las mujeres en situación de prostitución podían estar "infectadas” se realizaban operativos policiales durante los cuales se las detenía de manera legal, convirtiéndose la detención en ocasión para el abuso, la tortura y el asesinato.

Por último, y haciendo hincapié una vez más en la asociación entre “represión, policía y moralización”, queremos traer a colación la campaña que el jefe de policía Santuccione, anticipándose a la dictadura, impulsó respecto del carnaval semanas antes del golpe. A través de una resolución que tenía como objetivo "asegurar la moral y el orden público” durante carnaval, anunciaba que se "prohibía el uso de caretas y disfraces, banderas o insignias que afecten los intereses patrióticos así como vestiduras sacerdotales, policiales o militares” (Los Andes, 27/2/1976). También impedía cantos y danzas indecentes o que "por lo sugestivas e insinuantes choquen al sentimiento moral y costumbres del pueblo y su idiosincrasia” (Los Andes, 27/2/1976). La “prevención” de los desórdenes del carnaval fue una nueva excusa para que la policía provincial acentuara las razzias y las detenciones arbitrarias y masivas a días del golpe. De hecho uno de los jóvenes torturado y asesinado mencionado en este trabajo, Alaniz, fue secuestrado en un baile de carnaval (Los Andes, 27/2/1976).

\section{Cierre provisorio.}

Este breve recorrido por algunos casos de violencia institucional en la Mendoza pre dictatorial nos lleva a plantear algunas anticipaciones de sentido y preguntas.

En primer lugar, y retomando una vez más a Tiscornia cuando plantea que las construcciones normativas construidas en los bordes de la legalidad no están al margen de la ley sino que "son sistemas superpuestos y engarzados en las prácticas de castigo estatal”, entendemos que las formas punitivas que fueron parte de una estrategia represiva para esta época, sufrieron cierta homogeneización (Tiscornia, 2009:3). Esto llevó por un lado a la homologación de “delincuentes comunes y subversivos” a la hora de aplicar las prácticas de castigo estatal, al establecimiento de un mismo modus operandi, y a la creación de lugares que hicieron las veces de “depósitos de cuerpos”, tanto para unos como para otros. Por otro lado, entendemos que esto explicaría el aumento considerable de asesinatos de jóvenes de las clases populares en los meses previos al golpe. Quizás sea arriesgado plantearlo en estos términos, pero consideramos que la “caza de brujas” desplegada sobre los y las militantes, llevó a un 
crecimiento también de los “ajustes de cuentas” y “aprietes” policiales.

En segundo lugar, en la superposición de elementos tradicionales y novedosos dentro de la institución policial, en el marco del complejo proceso pre dictatorial, nos encontramos con un nuevo factor que hará las veces de “justificador” de ciertas acciones ilegales. Nos referimos a la dimensión “moralizadora” de la represión, y en particular a las bombas y asesinatos protagonizados por el Comando Pío XII. Es decir que la policía utilizó el carácter moralizador de la represión paraestatal, impulsado por sectores del nacionalismo católico, para “saldar algunas cuentas” con proxenetas, y de paso para acrecentar y aceitar su sistema de coimas hacia cabarets, ahora bajo la modalidad de colocación de bombas.

En tercer lugar, destacar que estas acciones represivas ilegales se articularon con procedimientos legales que otorgaron cierto marco jurídico al combate librado contra las “clases peligrosas”. Esto puede verse en significativas medidas impulsadas por el gobierno nacional, tales como la reforma del Código Penal, o leyes como la de Asociaciones Profesionales o Prescindibilidad; pero también en pequeñas disposiciones locales tales como la modificación del Código de Faltas o la reglamentación del carnaval. Y respecto de esto último, es importante enfatizar el hecho de que abordar estos procesos a escala local, prestando atención a las particularidades que adquirió la aplicación de la represión estatal en este caso en Mendoza, permite poner en cuestión algunos modelos explicativos elaborados en el centro del país, que muchas veces son traspolados a todo el territorio nacional.

Por último, estas primeras pinceladas sobre la relación entre violencia institucional y represión paraestatal en Mendoza apuntan a aportar al complejo mapa represivo argentino, que en los últimos años afortunadamente, se ha enriquecido con múltiples investigaciones.

\section{Notas}

1 Una versión preliminar de este trabajo fue presentado en las “IIas. Jornadas de Trabajo de la Red de Estudios sobre Represión y Violencia Política (RER)” realizadas en abril de 2016.

$\underline{2}$ En esta breve comunicación no realizamos un estado de la cuestión de la historia reciente de la represión, pero la bibliografía utilizada para trabajos anteriores y que han servido para reflexionar sobre este caso, pertenece a investigadores/as de la Red de Estudios sobre la Represión (https://redestudiosrepresion.wordpress.com/)

$\underline{3}$ La cobertura de estos asesinatos fue realizada por Los Andes bajo la dirección del célebre escritor Antonio Di Benedetto. Al respecto ver Rodríguez Agüero, 2016.

4 Código de Faltas, art. 54-54 bis, en Ley 3.365, Código de faltas de la Provincia de Mendoza, agosto de 2014. Disponible en web: http://www.saij.gob.ar/legislacion/ley-mendoza-3365-codigo faltas provincia mendoza.htm \%3Bjsessiodnid=zh7kc0dh16spqbfhtcta b09y?0

\section{Referencias bibliográficas}

Águila Gabriela (2013) “La represión en la historia reciente argentina: fases, dispositivos y dinámicas regionales”, en Águila Gabriela y Alonso Luciano (coords.), Procesos represivos y actitudes sociales: entre la España franquista y las dictaduras del Cono Sur, Prometeo, Buenos Aires.

Rodríguez Agüero Laura, Paredes Alejandro (2012). “Organizaciones de derecha y terrorismo de Estado. Mendoza 1970-1976”, en Revista de Estudios Trasandinos, volumen $17 \quad n^{\circ} 1$, en www.estudioshistoricos.ulagos.cl/. Chile, pp. 71 a 88.

Rodríguez Agüero, Laura (2013 a). “Violencia paraestatal y construcción del 'enemigo interno’. El caso de Mendoza (Argentina), 1973-1976”, en Revista A Contracorriente, volumen 11, n¹ 
Rodríguez Agüero, Laura (2013 b). “Centralización de la represión, violencia paraestatal y redes internacionales represivas en la Mendoza predictatorial”, en Sociohistórica, n 33, 2014 . Disponible en: http://www.sociohistorica.fahce.unlp.edu.ar/article/view/SH2014n33a02

Rodríguez Agüero, Laura (2016). “Los Andes, Antonio Di Benedetto y la violencia paraestatal. Mendoza, 19731976”, en Revista de Historia, N 17, pp. 122-144. Departamento de Historia, Facultad de Humanidades, Universidad Nacional del Comahue. Disponible http://revele.uncoma.edu.ar/htdoc/revele/index.php/historia/index

Tiscornia Sofia (2008). Activismo de los derechos humanos y burocracias estatales. El caso Walter Bulacio, Canadá, Editores del Puerto.

\section{Fuentes orales}

Entrevista realizada por Laura Rodríguez Agüero a Alberto Atienza, ex editor de la sección policiales del diario El Andino, Mendoza, marzo de 2008.

\section{Fuentes escritas}

“Grave denuncia en una fiscalía”, en Los Andes, Mendoza 19 de marzo de 1976.

“Aparente secuestro de un estudiante” en Los Andes, Mendoza, 22 de noviembre de 1975.

“Tres policías detenidos por intentar matar un chileno”, en Los Andes, Mendoza 13 de enero de 1976.

“Resolución policial sobre carnavales”, en Los Andes, 23 de marzo de 1976. 